\title{
Commutators and Self-Adjointness of Hamiltonian Operators
}

\author{
William G. Faris \\ Battelle Institute, Advanced Studies Center, Carouge-Geneva, Switzerland \\ Richard B. Lavine \\ Mathematics Department, University of Rochester, Rochester, N.Y., USA
}

Received September 10, 1973

\begin{abstract}
A time dependent approach to self-adjointness is presented and it is applied to quantum mechanical Hamiltonians which are not semi-bounded. Sufficient conditions are given for self-adjointness of Schrödinger and Dirac Hamiltonians with potentials which are unbounded at infinity. The method is the introduction of an auxiliary operator $N \geqq 0$ whose rate of change (commutator with the Hamiltonian) is bounded by a multiple of $N$.
\end{abstract}

\section{Introduction}

Let $\mathscr{H}$ be a Hilbert space and $H$ be a Hermitian operator acting in $\mathscr{H}$. That is, $H$ is a linear transformation (defined on a dense linear subspace $\mathscr{D}(H) \subset \mathscr{H}$ and taking values in $\mathscr{H})$ such that $\langle H f, g\rangle=\langle f, H g\rangle$ for all $f$ and $g$ in $\mathscr{D}(H)$.

The Schrödinger equation associated with $H$ is $i \frac{d u(t)}{d t}=H u(t)$.

The initial value problem with initial condition $u(0)=f$ has the formal solution $u(t)=\exp (-i t H) f$, but it is possible that the series expansion for the exponential does not converge for sufficiently many vectors in $\mathscr{D}(H)$ to determine a unitary operator $\exp (-i t H)$.

The exponential of a self-adjoint operator, however, is uniquely determined (by the spectral theorem). Thus any self-adjoint extension of $H$ leads to a solution of the initial value problem for the Schrödinger equation. The typical quantum mechanical Hamiltonian is a real operator (that is, it commutes with some conjugation), so it has selfadjoint extensions. The problem that remains is whether $H$ has a unique self-adjoint extension.

If $H$ is the sum of (positive) kinetic energy and potential energy terms, the Schrödinger equation describes a particle moving in configuration space under the influence of forces determined by the potential energy. If the potential energy is unbounded below, the kinetic energy 
of the particle may increase and the potential energy decrease so rapidly that the particle reaches a point where the potential is infinite. The particle must then be given directions on what to do next. In this case different "boundary conditions" (restrictions on the domain of the adjoint operator) determine different self-adjoint extensions. The same thing can happen if a strong repulsive force pushes the particle out to infinity in a finite amount of time.

If the potential energy is bounded below, the kinetic energy remains bounded and no such catastrophe can happen. Even if the potential energy is unbounded below in a small region, the uncertainty principle (more precisely, Sobolev inequalities) may prevent collapse. In this case, when $H$ is bounded below, there is at least a canonical self-adjoint extension, if not a unique one, the Friedrichs extension. And it can be shown in many cases that this operator is the only self-adjoint extension [8].

There are certain situations (such as an electron in a constant electric field) where, in spite of the fact that $H$ is not semi-bounded, the dynamics should be well determined. This can be shown by an analogue of an argument in classical mechanics. Let $u(t)$ be the solution of the Schrödinger equation and $N$ be a suitable positive operator which measures the magnitude of the momentum and the distance from a starting point. Write the expectation of $N$ as $n(t)=\langle u(t), N u(t)\rangle$. Then $\frac{d n(t)}{d t}$ $=\langle u(t), i[H, N] u(t)\rangle$. If the force doesn't grow too rapidly at infinity one can derive an inequality $\pm i[H, N] \leqq c N$. This says that $\pm \frac{d n(t)}{d t}$ $\leqq c n(t)$, and so $n(t) \leqq n(0) \exp (c|t|)$. If the particle starts off reasonably well localized, so that $n(0)$ is finite, then $n(t)$ remains finite for all $t$, the particle never reaches infinity, and no catastrophe occurs. The purpose of this note is to make this argument precise and to prove uniqueness of the self-adjoint extension for Hamiltonian operators satisfying such an inequality.

Our main mathematical result is Theorem 5 . It states roughly that self-adjointness for a Schrödinger operator which is bounded below implies self-adjointness for the operator plus a potential which decreases at most quadratically at infinity. (This new operator of course need not be bounded below.) A typical application is given in Corollary 5.1.

The result is a consequence of a variant of an abstract theorem of Nelson [9] on commutators and self-adjointness. The hypothesis of Nelson's theorem is a first order estimate on a commutator. His theorem extends a theorem of Glimm and Jaffe [6] which requires a second order estimate on the commutator. All these authors were concerned with a 
different application of commutator theorems, to quantum fields. In our applications only one operator is given and one must make a suitable choice of the auxiliary operator $N$ in order to obtain the estimates.

Our other main contribution is a rigorous formulation of the intuitive physical picture of the particle never moving so fast as to reach infinity in finite time. This formulation is given in Theorems 2 and 3 . However for applications the original abstract theorem (Theorem 1) is sufficient. We also give a new proof of this theorem and an application to Dirac operators (Theorem 6).

The problem for the Schrödinger equation in the one-dimensional case has been previously treated by examining the asymptotic behavior of solutions of an ordinary differential equation [2; Chapter 13, $\S 6$, Corollaries 17 and 22]. The correspondence of these results with the time required to reach infinity in classical mechanics has been noted by Wightman [12]. (This correspondence is not exact; some of the borderline cases are quite delicate, due to quantum mechanical effects $[10,3,4]$.)

The question of essential self-adjointness of the $n$-dimensional Schrödinger operator has a long history (see [11] and the references there). The definitive treatment is due to Kato [8]; he makes use of an inequality involving absolute values of wave functions. For the Dirac operator Chernoff [1] has recently given an essential self-adjointness result which has a very clear physical significance. He argues in effect that since nothing can travel faster than the speed of light (in a relativistic theory such as the Dirac theory), it is impossible to reach infinity in a finite time.

\section{Essential Self-Adjointness and Invariant Domains}

Nelson's commutator theorem has to do with the essential selfadjointness of a Hermitian operator. If $H$ is a Hermitian operator and $H_{1}$ is a self-adjoint extension of $H$, then $H \subset \bar{H} \subset H_{1} \subset H^{*}$, where $\bar{H}$ is the closure and $H^{*}$ is the adjoint (and the inclusions are between graphs). The operator $H$ is said to be essentially self-adjoint if its closure $\bar{H}$ is self-adjoint. Since then $\bar{H}=(\bar{H})^{*}=H^{*}$, it follows that $H_{1}=\bar{H}$. Thus if $H$ is essentially self-adjoint it has a unique self-adjoint extension. (The converse is also true.)

We denote the domain of an operator $A$ by $\mathscr{D}(A)$, and if $N$ is positive and self-adjoint we call $\mathscr{D}\left(N^{\frac{1}{2}}\right)=\mathscr{2}(N)$ (the domain of the quadratic form of $N)$. The following result is closely related to a theorem of Nelson [9]. 
Theorem 1. Let $H$ be a Hermitian operator and $N \geqq 0$ a positive selfadjoint operator satisfying

(i) $\mathscr{D}(N) \subset \mathscr{D}(H)$.

(ii) $\pm i[H, N] \leqq c N$ for some constant $c<\infty$.

Then $H$ is essentially self-adjoint.

The quadratic form inequality (ii) in the statement of the theorem means that $\pm i\{\langle H f, N f\rangle-\langle N f, H f\rangle\} \leqq c\langle f, N f\rangle$ for all $f$ in $\mathscr{D}(N)$.

Proof. Without loss of generality we may assume that $N \geqq 1$. We use the fact that $H$ is essentially self-adjoint provided that the range of $H-d i$ is dense in $\mathscr{H}$ whenever $|d|$ is sufficiently large.

Let $f$ be orthogonal to the range of $H-d i$. Then in particular $\operatorname{Im}\left\langle f,(H-d i) N^{-1} f\right\rangle=0$. It follows that

$$
\begin{aligned}
2 d\left\langle f, N^{-1} f\right\rangle & =i\left\{\left\langle H N^{-1} f, f\right\rangle-\left\langle f, H N^{-1} f\right\rangle\right\} \\
& =\left\langle N^{-1} f, i[H, N] N^{-1} f\right\rangle .
\end{aligned}
$$

Hence $\pm 2 d\left\langle f, N^{-1} f\right\rangle \leqq c\left\langle f, N^{-1} f\right\rangle$. If $2|d|>c$, this implies that $f=0$.

Corollary 1.1. Let $H$ be a Hermitian operator and $N \geqq 0$ a positive self-adjoint operator. Let $\mathscr{C}$ be a core for $N$ (a dense linear subspace of the Hilbert space $\mathscr{D}(N)$ ) such that $\mathscr{C} C \mathscr{D}(H)$. Assume that there hold estimates

(i) $\|H f\|^{2} \leqq a\|N f\|^{2}+b\|f\|^{2}, f \in \mathscr{C}$.

(ii) $\pm i[H, N] \leqq c N$ (as quadratic forms on $\mathscr{C})$.

Then $H$ is essentially self-adjoint. (In fact, even its restriction to $\mathscr{C}$ is essentially self-adjoint.)

Proof. We have $\mathscr{D}(N) \subset \mathscr{D}(\bar{H})$, by (i), and (ii) implies that $\pm i[\bar{H}, N] \leqq c N$ as quadratic forms on $\mathscr{D}(N)$, because both forms can be approximated using vectors in $\mathscr{C}$. Thus Theorem 1 applies to $\bar{H}$.

The advantage of this result over that of Nelson is that there is more flexibility in the choice of the core. This is needed for our main application (Theorem 5).

The following two results provide a time-dependent interpretation of Theorem 1. They may be used together to prove uniqueness of the self-adjoint extension.

Theorem 2. Let $H$ and $N$ satisfy the conditions of Theorem 1, and let $H_{1}$ be a self-adjoint extension of $H$. Then $\mathscr{2}(N)$ is invariant under $\exp \left(-i t H_{1}\right)$.

Proof. We must show that any solution of the Schrödinger equation with initial value in $\mathscr{2}(N)$ remains in $\mathscr{2}(N)$ for all time. We will do this by showing that if $u(t)=\exp \left(-i t H_{1}\right) u$, we have the inequality $\langle u(t), N u(t)\rangle$ $\leqq\langle u, N u\rangle \exp (c|t|)$. 
First let $u \in \mathscr{D}\left(H_{1}\right)$ and consider $N_{\varepsilon}=N(\varepsilon N+1)^{-1}$. Then

$$
\begin{aligned}
\frac{d}{d t}\left\langle u(t), N_{\varepsilon} u(t)\right\rangle & =\left\langle u(t), i\left[H_{1}, N_{\varepsilon}\right] u(t)\right\rangle \\
& =\left\langle(\varepsilon N+1)^{-1} u(t), i[H, N](\varepsilon N+1)^{-1} u(t)\right\rangle
\end{aligned}
$$

which has absolute value bounded by $c\left\langle u(t), N_{\varepsilon} u(t)\right\rangle$. We may integrate the inequality to obtain $\left\langle u(t), N_{\varepsilon} u(t)\right\rangle \leqq\left\langle u, N_{\varepsilon} u\right\rangle \exp (c|t|)$.

This estimate holds for all $u$ in $\mathscr{H}$ because $\mathscr{D}\left(H_{1}\right)$ is dense in $\mathscr{H}$ and $N_{\varepsilon}$ is bounded. Now let $u \in \mathscr{Z}(N)$. Taking limits as $\varepsilon \rightarrow 0$ gives $\langle u(t), N u(t)\rangle \leqq\langle u, N u\rangle \exp (c|t|)$.

Theorem 3. Let $H$ be a Hermitian operator and let $N \geqq 0$ be a selfadjoint operator with $\mathscr{D}(N) \subset \mathscr{D}(H)$. Then $H$ has at most one self-adjoint extension $H_{1}$ such that $\exp \left(-i t H_{1}\right)$ leaves $2(N)$ invariant.

Proof. We assume without loss of generality that $H$ has a selfadjoint extension and that $N \geqq 1$. First we show that there is a unique continuous Hermitian form $h(f, g)$ on $\mathscr{Z}(N)$ such that $\langle f, H g\rangle=h(f, g)$ for $g$ in $\mathscr{D}(N)$. Let $K$ be a self-adjoint extension of $H$. Then $\|K f\|$ $\leqq c\|N f\|$, by the closed graph theorem. That is, $K^{2} \leqq c^{2} N^{2}$, which implies [5] that $|K| \leqq c N$. Thus $|\langle f, K g\rangle| \leqq\left\||K|^{\frac{1}{2}} f\right\|\left\||K|^{\frac{1}{2}} g\right\|\left\langle c\left\|N^{\frac{1}{2}} f\right\|\right.$ - $\left\|N^{\frac{1}{2}} g\right\|$. Hence the form $\langle f, H g\rangle$ extends to a continuous form on $\mathscr{Q}(N)$.

Next we use this form to define a Hermitian operator $\hat{H}$ which extends $H$. For all $g$ in $\mathscr{2}(N)$ such that there exists $u$ in $\mathscr{H}$ with $\langle f, u\rangle$ $=h(f, g)$, let $\hat{H} g=u$. (The operator $\hat{H}$ is something like a Friedrichs extension of $H$, but of course $H$ need not be semi-bounded.)

Let $H_{1}$ be a self-adjoint extension of $H$ such that $\exp \left(-i t H_{1}\right)$ leaves $\mathscr{2}(N)$ invariant. Consider $g \in \mathscr{E}=\mathscr{D}\left(H_{1}\right) \cap \mathscr{L}(N)$. If $f \in \mathscr{D}(N)$, then $\left\langle f, H_{1} g\right\rangle=\langle H f, g\rangle=h(f, g)$, so $g \in \mathscr{D}(\hat{H})$ and $\hat{H} g=H_{1} g$. Thus $\hat{H}$ extends the restriction of $H_{1}$ to $\mathscr{E}$.

Next observe that since $\mathscr{E}$ is dense in $\mathscr{H}$ and $\exp \left(-i t H_{1}\right)$ leaves $\mathscr{E}$ invariant, $\mathscr{E}$ is a core for $H_{1}$. (This is an easy consequence of the spectral theorem and Fourier analysis.) Since $\hat{H}$ extends $H_{1}$ restricted to $\mathscr{E}$, the closure of $\hat{H}$ must extend $H_{1}$. But a self-adjoint operator has no proper Hermitian extensions. So the closure of $\hat{H}$ must equal $H_{1}$. This argument holds for any such self-adjoint extension, so all such extensions must be equal.

\section{Schrödinger Operators}

In our applications of Theorem 1 the operator $H$ is the Hamiltonian and we must choose a suitable auxiliary operator $N$.

For Schrödinger operators the Hilbert space is $\mathscr{H}=L^{2}\left(\mathbb{R}^{n}, d x\right)$. The self-adjoint operators $p_{i}=-i \partial / \partial x_{i}$ and $q_{j}=$ multiplication by $x_{j}$ 
act in $\mathscr{H}$. First we take $N=p^{2}+q^{2}$, where $p^{2}=-\Delta$ and $q^{2}$ is multiplication by $x^{2}$. We shall often use the fact that $N \geqq 1$. Note that the set of finite linear combinations of Hermite functions is a core for $N$, and therefore so is the Schwartz space $\mathscr{S}$ (of test functions for tempered distributions). We shall also need to estimate various operators in terms of $N$.

Proposition 1. For $f$ in $\mathscr{D}(N),\left\|p^{2} f\right\|^{2}+\left\|q^{2} f\right\|^{2} \leqq\|N f\|^{2}+c\|f\|^{2}$.

Proof. It is sufficient to make the estimate for $f$ in some nice core of $N$. We use the double commutator argument of Jaffe and obtain the quadratic form estimate

$$
\begin{aligned}
N^{2} & =p^{4}+q^{4}+p^{2} q^{2}+q^{2} p^{2} \\
& =p^{4}+q^{4}+\sum_{j=1}^{n} 2 q_{j} p^{2} q_{j}+\left[q_{j},\left[q_{j}, p^{2}\right]\right] \geqq p^{4}+q^{4}-2 n .
\end{aligned}
$$

Thus we may take $c=2 n$.

Let $v$ be a real measurable function on $\mathbb{R}^{n}$. Then $p^{2}=-\Delta$ and $v(q)$ $=$ multiplication by $v$ are self-adjoint operators acting in $\mathscr{H}=L^{2}\left(\mathbb{R}^{n}, d x\right)$. Set $H=p^{2}+v(q)$, defined on the intersection of the domains of $p^{2}$ and $v(q)$. If $v$ is locally in $L^{2}$, then $H$ is densely defined and so $H$ is a Hermitian operator.

Theorem 4. Let $v$ be a real continuous function on $\mathbb{R}^{n}$ such that $|\nabla v(x)| \leqq a|x|+b$ for some constants $a$ and $b$. Then the Schrödinger operator $H=p^{2}+v(q)$ is essentially self-adjoint.

Observe that $H$ is real, so self-adjoint extensions exist. The only question is that of uniqueness.

Proof. We write $v^{\prime}$ for the gradient $v^{\prime}=\nabla v$. The hypothesis of the theorem is that $v$ is a continuous function whose partial derivatives $\partial v / \partial x_{j}$ are measurable functions, and that $\left|v^{\prime}(x)\right| \leqq a|x|+b$. It follows that $|v(x)| \leqq \frac{1}{2} a x^{2}+b|x|+v(0) \leqq c x^{2}+d$.

We intend to apply Corollary 1.1 , taking $\mathscr{C}=\mathscr{S}$ and $N=p^{2}+q^{2}$. First we note that if $f \in \mathscr{S}$, then $\left(1+x^{2}\right) f \in L^{2}$, so $v f \in L^{2}$. It follows that $\mathscr{S} \subset \mathscr{D}(H)$.

Next we observe that since $v$ satisfies an estimate $v(x)^{2} \leqq r x^{4}+s$, we have $\|v(q) f\|^{2} \leqq r\left\|q^{2} f\right\|+s\|f\|^{2}$. Thus by Proposition 1, hypothesis (i) of Corollary 1.1 is satisfied.

The bound on the gradient of $v$ implies that if $f \in \mathscr{S}$, then $\frac{\partial}{\partial x_{i}}(v f) \in L^{2}$. Thus the following commutator calculations are justified. 
The expressions occurring in the calculations are of course to be interpreted as quadratic forms. The result is

$\pm i[H, N]= \pm i\left\{\left[p^{2}, q^{2}\right]+\left[v(q), p^{2}\right]\right\}$

$= \pm\left\{2(p \cdot q+q \cdot p)-\left(p \cdot v^{\prime}(q)+v^{\prime}(q) \cdot p\right)\right\}$

$\leqq 2\left(p^{2}+q^{2}\right)+p^{2}+\left(v^{\prime}(q)\right)^{2} \leqq 2\left(p^{2}+q^{2}\right)+p^{2}+2\left(a^{2} q^{2}+b^{2}\right) \leqq c N$

for some constant $c$. Thus hypothesis (ii) of Corollary 1.1 is also satisfied. The conclusion follows.

Notice that we have even shown that the restriction of $H$ to $\mathscr{S}$ is essentially self-adjoint, which is more than is stated in the theorem.

Theorem 4 gives the limiting case for Schrödinger operators in the sense that without a quadratic bound on the negative part of $v$ the conclusion may fail [2; Chapter 13, §6, Corollary 22]. (The particle rushes to infinity in a finite time.) However the extensive information available in the case $n=1[10,3,4]$ shows that it is far from being the sharpest condition.

Kato [8] has recently given an argument that shows that positive potentials do not usually lead to problems with essential self-adjointness. His basic result is that if $v \geqq 0$ and is locally in $L^{2}$, then $p^{2}+v(q)$ is essentially self-adjoint on the space of $C^{\infty}$ functions with compact support. The argument is based on the lemma: If $f$ is real and $f$ and $\Delta f$ are locally in $L^{1}$, then $\Delta|f| \geqq \Delta f \operatorname{sign} f$ as distributions. It goes as follows. If $f$ is a real function in $L^{2}$ which is orthogonal to the range of $p^{2}+v(q)+1$, then $(-\Delta+v+1) f=0$ in the sense of distributions. Thus $\Delta f=(v+1) f$ is locally in $L^{1}$, so by Kato's lemma $\Delta|f| \geqq \Delta f \operatorname{sign} f$ $=(v+1) f \operatorname{sign} f=(v+1)|f| \geqq|f|$. Hence $(1-\Delta)|f| \leqq 0$. But $(1-\Delta)^{-1}$ acts on the space of tempered distributions and preserves positivity, so $|f| \leqq 0, f=0$. This proves essential self-adjointness.

Kato was able to extend this result to potentials which are unbounded below. Our next theorem will provide an alternative method to extend it to such potentials. The method depends only on the essential selfadjointness result for positive potentials and not on how it was obtained. (One can easily imagine other applications where $v$ is not even locally in $L^{2}$.)

First we make a preliminary remark about local singularities. Certain unbounded negative potentials may be dealt with quite simply by an extension [5] of a lemma of Davies. The lemma states that if $v \geqq 0$ and $p^{2}+v(q)$ is essentially self-adjoint on some linear subspace $\mathscr{C}$, and if $w$ is a real function such that $(w(q))^{2} \leqq a^{2}\left(p^{2}+b\right)^{2}$, then $(w(q))^{2}$ $\leqq a^{2}\left(p^{2}+v(q)+b\right)^{2}$. In particular, if the first estimate holds for some $a^{2}<1$, then so does the second, and so $p^{2}+v(q)+w(q)$ is also essentially self-adjoint on $\mathscr{C}$. [It follows from Sobolev inequalities that if $w \in L^{p}$ for 
some $p \geqq n / 2, p \geqq 2$ (and $p>2$ when $n=4$ ), then for all $a>0$ there exists $b<\infty$ such that the first estimate holds.]

We shall now show how one can deduce a result allowing quadratic decrease at infinity from these results and our theory.

Theorem 5. Let $v$ and $w$ be real measurable functions on $\mathbb{R}^{n}$ such that $-v(x) \leqq c x^{2}+d$ and such that $-w(q) \leqq a p^{2}+b$ (as quadratic forms) for some $a<1$. Let $H=p^{2}+v(q)+w(q)$. Let $\mathscr{C}$ be a linear subspace of $\mathscr{D}(H)$ which is invariant under the $p_{k}$ and $q_{j}$. Assume that $H+2 c q^{2}$ is essentially self-adjoint on $\mathscr{C}$. Then $H$ is essentially self-adjoint on $\mathscr{C}$.

Proof. First note that $H+c q^{2} \geqq(1-a) p^{2}-b-d$. Hence by adding a constant to $H$ we may ensure that $H+c q^{2} \geqq(1-a) p^{2} \geqq 0$.

We choose $N$ as the closure of $H+2 c q^{2}$ and apply Corollary 1.1. We must verify that $\|H f\|^{2} \leqq\|N f\|^{2}+k\|f\|^{2}$ for $f$ in $\mathscr{C}$. We calculate that

$$
\begin{aligned}
N^{2} & =H^{2}+4 c^{2} q^{4}+2 c\left(q^{2} H+H q^{2}\right) \\
& =H^{2}+4 c^{2} q^{4}+4 c \sum_{j} q_{j} H q_{j}+2 c \sum_{j}\left[q_{j},\left[q_{j}, H\right]\right] \\
& =H^{2}+4 c \sum_{j} q_{j}\left(H+c q^{2}\right) q_{j}-4 c n \geqq H^{2}-4 c n
\end{aligned}
$$

in the sense of quadratic forms on $\mathscr{C}$. (Here we have evaluated the double commutator as in Proposition 1.) This is the required estimate (with $k=4 c n$ ).

To check the other condition we must estimate $i[H, N]$. We have

$$
\begin{aligned}
& \pm i[H, N]= \pm i\left[p^{2}, 2 c q^{2}\right]= \pm 4 c(p \cdot q+q \cdot p) \leqq 4 c\left(p^{2}+q^{2}\right) \\
& \quad \leqq 4 c(1-a)^{-1}\left(H+c q^{2}\right)+4 c q^{2} \leqq k\left(H+c q^{2}\right)+k c q^{2}=k N
\end{aligned}
$$

for some $k$ sufficiently large. This is the other estimate.

Corollary 5.1 [8]. Let $v$ and $w$ be real functions such that $v(x)$ $\geqq-b x^{2}-c$ and is locally in $L^{2}$, and such that $w$ is in $L^{p}$ for some $p \geqq n / 2$, $p \geqq 2(p>2$ when $n=4)$. Then $H=p^{2}+v(q)+w(q)$ is essentially selfadjoint.

Proof. Let $\mathscr{C}$ consist of the $C^{\infty}$ functions with compact support. Then by the cited results $N=H+2 c q^{2}=p^{2}+\left(v(q)+2 c q^{2}\right)+w(q)$ is essentially self-adjoint on $\mathscr{C}$.

It follows from the fact that $w(q)^{2} \leqq a^{2}\left(p^{2}+b\right)^{2}$ that $-w(q) \leqq|w(q)|$ $\leqq a\left(p^{2}+b\right)$ [5]. Thus the quadratic form estimate is also satisfied. So we have proved that $H$ is essentially self-adjoint on the space of $C^{\infty}$ functions with compact support.

Notice that this corollary is much better than Theorem 4; in particular there is no condition on the derivative of $v$. However it uses more machinery. 


\section{Dirac Operators}

Let $\mathscr{H}=L^{2}\left(\mathbb{R}^{3}, d x ; \mathbb{C}^{4}\right)$, the space of square integrable functions on $\mathbb{R}^{3}$ with values in $\mathbb{C}^{4}$. The quantum mechanical operators $p_{i}$ and $q_{j}$ act in $\mathscr{H}$ in the natural way. The free Dirac operator $\alpha \cdot p+\beta$ is a selfadjoint operator acting in $\mathscr{H}$. Here $\alpha_{1}, \alpha_{2}, \alpha_{3}$, and $\beta$ are certain 4 by 4 Hermitian matrices. They act on $\mathbb{C}^{4}$ and hence on $\mathscr{H}$ in the obvious way. The matrices $\alpha_{j}$ have the property that if $k \in \mathbb{R}^{3}$, then $(\alpha \cdot k)^{2}=k^{2}$. It follows immediately that $\mathscr{D}(\alpha \cdot p+\beta)=\mathscr{D}(\alpha \cdot p)=\mathscr{D}(|p|)$.

Let $v$ be a real measurable function on $\mathbb{R}^{3}$. Then $v(q)$ is a self-adjoint operator acting in $\mathscr{H}$. If $v$ is locally in $L^{2}$, then $H=\alpha \cdot p+\beta+v(q)$ is a (densely defined) Hermitian operator. This is the Dirac Hamiltonian.

As in the case of the Schrödinger Hamiltonians it is immediate that self-adjoint extensions of $H$ exist. In fact $H$ is real with respect to the conjugation $J$ which reverses the direction of time. (This is defined as follows. There exists a 4 by 4 matrix $\tau$ such that $\tau \bar{\alpha}_{i}=-\alpha_{i} \tau$ and $\tau \bar{\beta}=\beta \tau$. The conjugation $J$ is given by $J f=\tau \bar{f}$. It is easy to see that $H$ commutes with $J$.)

Theorem 6. Let $v$ be a real continuous function on $\mathbb{R}^{3}$ such that $|\nabla v(x)| \leqq a \exp (k|x|)$ for some constants $a$ and $k$. Then the Dirac operator $H=\alpha \cdot p+\beta+v(q)$ is essentially self-adjoint.

Proof. The potential $v$ also satisfies an exponential bound $|v(x)|$ $\leqq|v(0)|+a / k \exp (k|x|)$. So this time we choose $N$ as the closure of the operator $p^{2}+w(q)$, where $w(x)=\exp (2 k|x|)$. Since $w \geqq 0$, it follows from Kato's theorem cited above that $N$ is a positive self-adjoint operator and that the space of $C^{\infty}$ functions with compact support is a core $\mathscr{E}$ for $N$. It is clear that $\mathscr{C} \subset \mathscr{D}(H)$. We now apply Corollary 1.1. Since $\beta$ is bounded, it is sufficient to work with $H=\alpha \circ p+v(q)$ and verify the two hypotheses.

For the first we need only a trivial first order estimate, since $H^{2} \leqq 2\left(p^{2}+v(q)^{2}\right) \leqq c\left(p^{2}+w(q)\right)+d=c N+d \leqq a N^{2}+b$.

We now show that the other hypothesis is also satisfied, that is, that $\pm i[H, N] \leqq c N$ as quadratic forms on $\mathscr{C}$. We write $v^{\prime}=\nabla v$ and compute

$$
\begin{aligned}
\pm i[H, N] & = \pm i\left\{[\alpha \cdot p, w(q)]+\left[v(q), p^{2}\right]\right\} \\
& = \pm\left\{\alpha \cdot w^{\prime}(q)-\left(p \cdot v^{\prime}(q)+v^{\prime}(q) \cdot p\right)\right\} \leqq\left|w^{\prime}(q)\right|+p^{2}+\left(v^{\prime}(q)\right)^{2} \\
& \leqq 2 k w(q)+p^{2}+a^{2} w(q) \leqq c\left(p^{2}+w(q)\right)=c N .
\end{aligned}
$$

Thus we have established that $H$ is essentially self-adjoint on the space of $C^{\infty}$ functions with compact support.

Even though this result allows exponential growth, it is not the sharpest result one would expect for the Dirac equation. In fact Chernoff [1] has shown using hyperbolicity that for smooth $v$ no growth 
restrictions at infinity are needed. (He has remarked to us that his result holds for arbitrary continuous $v$, since any continuous function is the sum of a smooth function and a bounded function.)

We thank Professor Charles McCarthy for helpful comments.

\title{
References
}

1. Chernoff, P. R.: J. Functional Anal. 12, $401-414$ (1973)

2. Dunford, N., Schwartz, J.T.: Linear operators, Part II. New York: Interscience 1963

3. Eastham, M.S.P.: Bull. London Math. Soc. 4. 340-344 (1972)

4. Eastham, M.S.P.: Quart. J. Math. (Oxford) (2) 24, 257-263 (1973)

5. Faris, W.: Commun. math. Phys. 30, 23-34 (1973)

6. Glimm, J., Jaffe, A. : J. Math. Phys. 13, 1568-1584 (1972)

7. Kato, T.: J. Functional Anal. 12, 415-417 (1973)

8. Kato, T.: Israel J. Math. 13, 135-148 (1972)

9. Nelson, E. : J. Functional Anal. 11, 211-219 (1972)

10. Rauch, J., Reed, M.: Commun. math. Phys. 29, 105-111 (1973)

11. Wienholtz, E.: Arch. Math. 10, 126-133 (1959)

12. Wightman,A.S.: The problem of existence of solutions in quantum field theory, in Proceedings Fifth Annual Eastern Theoretical Physics Conference, ed. by D. Feldman. New York: Benjamin 1967

\author{
W. G. Faris \\ Battelle Institute \\ Advanced Studies Center \\ CH-1227 Carouge-Geneva \\ Switzerland \\ R. B. Lavine \\ Mathematics Department \\ University of Rochester \\ Rochester, N.Y. 14627 \\ USA
}

